\title{
¿El armario está abierto?: estudios sobre diversidad sexual en El Salvador
}

Amaral Palevi Gómez Arévalo'

'Universidade Federal de Pernambuco (UFPE), Recife/PE - Brasil

RESUMEN - ¿El armario está abierto?: estudios sobre diversidad sexual en El Salvador. El presente texto tiene por objetivo analizar 39 producciones universitarias sobre diferentes temáticas de diversidad sexual producidas entre 1988 a 2015, las cuales se organizan en 4 ejes/binomios: Prácticas sexuales/Cuerpos, Matrimonio/Familias, Identidades/Prejuicios y Derechos/ Ciudadanías. Al mismo tiempo se proponen nuevas temáticas para investigar y se reflexiona sobre los retos al interior de la educación superior salvadoreña para constituir un campo de Estudios sobre Diversidad Sexual. Concluyendo, a pesar de la existencia dispersa de 39 producciones universitarias, las cuales son un avance, el armario de la academia salvadoreña está aún cerrado, y por tal motivo surge la pregunta ¿cómo lo podemos abrir? Palabras-clave: El Salvador. Educación Superior. Universidades. Sexualidad. Género.

ABSTRACT - Is the closet opened?: sexual diversity studies in El Salvador. This paper aims to analyze 39 academic papers on different themes about sexual diversity produced 1988-2015. The analysis is based on four pairs: Sexual practices/Bodies, Marriage/Families, Identities/Prejudice and Rights/ Citizenship. Based on that, we propose new themes for investigation and reflections about the challenges within the Salvadoran higher education to build the field of Sexual Diversity Studies. In conclusion, despite the existence of 39 papers - which are a step forward -, the closet of the Salvadoran Academy is still closed, and therefore a question arises: how can we open it? Keywords: El Salvador. Higher Education. Universities. Sexuality. Gender.

Educação \& Realidade, Porto Alegre, v. 42, n. 4, p. 1375-1397, oct./dic. 2017. http://dx.doi.org/10.1590/2175-623662013 


\section{La Provocación: a manera de introducción}

A la razón de una investigación sobre la estructuración e historia del movimiento organizado de Diversidad Sexual en El Salvador y a la vista que las principales fuentes de información documentales que estaba ocupando eran los informes sobre violación a los derechos humanos que producen, principalmente, las diferentes asociaciones, organizaciones y colectivos de lesbianas, gays, bisexuales, transgéneros, transexuales, travestis e intersexuales (LGBTI) me surgió la interrogante de cuál era el papel de las universidades en la producción de conocimiento sobre diversidad sexual en el país, preguntándome específicamente: ¿Existen Estudios sobre Diversidad Sexual en las universidades de El Salvador? Tomando en cuenta que el contexto salvadoreño aún mantiene tabúes arraigados sobre la sexualidad, en donde el binarismo sexual heteronormativo es el guion que marca la vida en la sociedad y lo cual en muchas oportunidades se refleja al interior de la vida académica en las diferentes universidades. La respuesta a la pregunta anterior sería con total claridad que no existen ese tipo de estudios en El Salvador. Pero, a tal respuesta absoluta me resistí a considerarla como incuestionable.

Luego de realizar una primera investigación en los portales virtuales de las diferentes bibliotecas de las universidades, comencé a encontrar títulos de tesis de grados - en su mayoría - y algunas tesis de maestría que hacían referencias a identidades homosexuales, lesbianas y transexuales; además del estudio de fenómenos sociales como la homofobia, el matrimonio entre personas del mismo sexo, el derecho a la identidad, trabajo y diversidad sexual, entre otros de los 39 títulos de tesis producidas desde 1988 hasta el año 2015.

Tomando en cuenta ese contexto, este artículo tiene dos finalidades; por una parte, analizar los ejes en los cuáles se han organizado estas incipientes y esporádicas investigaciones sobre diversidad sexual; y su segunda finalidad es proponer líneas de investigación, retomando lo existente, para estructurar un bosquejo inicial de un campo que se pueda denominar como Estudios sobre Diversidad Sexual en El Salvador.

Para dar cuenta de lo anterior, este artículo se estructura en cuatro apartados. El primero de ellos se titula: Los hallazgos, el cual contiene una breve descripción de los materiales y producciones académicas universitarias elaboradas desde el año 1988 hasta el año 2015. Para su análisis se propone realizarlo por medio de la organización de binomios-ejes, a saber: Prácticas sexuales/Cuerpos, Matrimonio/Familias, Identidades/Prejuicios y Derechos/Ciudadanías. En el segundo apartado, titulado: La propuesta, se fortalecen los ejes temáticos anteriores con nuevas propuestas de temáticas de investigación para profundizar la comprensión y el análisis de la diversidad sexual desde una mirada académica. En el tercer apartado titulado: Los retos, se presentarán algunos de los desafíos que tienen este tipo Estudios al interior de la Educación Superior Salvadoreña. Para finalizar, en las palabras de cierre, se presentan las conclusiones del artículo.

1376 Educação \& Realidade, Porto Alegre, v. 42, n. 4, p. 1375-1397, oct./dic. 2017. 


\section{Los Hallazgos: producciones universitarias sobre diversidad sexual}

Investigar y recolectar producciones universitarias sobre diversidad sexual en las diferentes universidades de El Salvador ha sido un trabajo arqueológico (Foucault, 1972). En un primer momento consideraba categóricamente que no existía ningún tipo de producción universitaria sobre estos temas. Esta suposición se fundamenta en que El Salvador a diferencia de otros país como México que en el año 1998 inician los Estudios de la Diversidad Sexual al interior del Programa Universitario de Estudios de Género de la UNAM (Careaga; Cruz, 2004); en Brasil comenzaron a ser abordadas temáticas sobre sexualidad desde 1982 con el Núcleo de Estudos Populacionais (NEPO), pasando por la creación en 1993 del Núcleo de Estudos de Gênero (PAGU) hasta la creación del Centro Latinoamericano de Sexualidad y Derechos Humanos (CLAM) en el año 2002 por mencionar sólo tres ejemplos (Carrara, 2010); en Estados Unidos los estudios Lésbicos-Gay surgen en la década de 1970 (Hernández, 2004); en Cuba con la creación de la Sociedad Cubana Multidisciplinaria de Estudios de la Sexualidad (SOCUMES) en 1985 y el Centro Nacional de Educación Sexual (CENESEX) se fortalecen los estudios de la sexualidad emprendidos desde 1972 (Guerra; Echeverría; Bombino, 2013); o en el caso de Colombia que desde la década de 1990 convierte al homosexual en sujeto de estudio (Guerrero; Sutachan, 2012). Esa tradición académica no es evidente en El Salvador, a la fecha no se conocen núcleos, centros o líneas de investigación al interior de las universidades o de los programas de posgrados que traten sobre diversidad sexual.

No obstante, me resistí a aceptar que las universidades de El Salvador no hubieran desarrollado algún trabajo de grado o tesis de maestría que su eje central de discusión fueran temáticas sobre diversidad sexual. Ante tal situación me planteé realizar una indagación virtual en las diferentes bibliotecas universitarias tanto públicas como privadas. Colocando palabras claves en los motores de búsqueda como: gay, homosexual, sexualidad, lesbiana, bisexual, transexual, transgénero, travestí, sexual, VIH, Sida, entre otras. En muchas universidades no se generó ningún resultado, pero en otras comenzaron a surgir temáticas como prácticas homosexuales en la población masculina, discriminación a las minorías homosexuales en el trabajo informal, matrimonio homosexual, caracterización psicológica de mujeres lesbianas, educandos con tendencia homosexual, prácticas de feminización en transgéneros, entre otras. En total surgieron ante mí 28 referencias de producciones universitarias entre trabajos de grado y tesis de maestría. En algunas de las universidades no se encontraba disponibles sus catálogos en línea, por lo cual desconocía la existencia o no de producciones universitarias sobre estas temáticas. Con esta información base emprendí la tarea de realizar una visita de campo para procurar nuevas producciones en universidades que no disponían sus catálogos en línea, además tuve la colaboración de amigos para obtener copias de algunos trabajos de grado cuando estos no se encontraban digitalizados. Así se 
lograron recuperar 35 referencias bibliográficas de trabajos de grado y cuatro tesis de posgrado a nivel de maestría.

Entrando a las características generales de las producciones académicas, respecto a la temporalidad vemos que la primera producción data de 1988. Esta situación es interesante de hacerlo notar, ya que en esa misma década El Salvador vivía uno de sus episodios históricos más obscuros, como lo fue la guerra interna. Después tendrían que pasar diez años para que la segunda producción universitaria saliera a la luz. Al cerrar la década de 1990 se contaban con 2 producciones universitarias. En la década del 2000 las producciones comienzan a surgir de manera esporádica pero en mayor número que las décadas pasadas. A la finalización de la década de 2000 se produjeron 14 producciones académicas. Ahora en la mitad de la década del 2010, se ha experimentado un incremento que se puede caracterizar como vertiginoso, ya que en la razón de cinco años se han producido 22 investigaciones sobre temáticas de diversidad sexual. En otras palabras, en solamente cinco años se logró superar el número de producciones universitarias que en los veintidós años anteriores. ¿Algo estará mudando?

Colocando la mirada en las áreas disciplinares que donde fueron creadas estas producciones universitarias, nos encontramos que una mayoría (13) fueron producidas desde una mirada de la Psicología. Las Ciencias Jurídicas con 11 producciones ocupa la segunda posición. La tercera área de conocimiento ha sido la Comunicación Social con un total de 6. Luego la Maestría en Derechos Humanos y Educación para la Paz tiene la producción de 2 tesis con una diferencia de 17 años entre ellas, y la Maestría en Servicios Integrales en Salud Sexual y Reproductiva también con 2 tesis. Para culminar las áreas de conocimiento de Educación, Antropología Sociocultural, Trabajo social, Medicina y Salud Materno-Infantil con una producción académica cada una.

Al colocar la mirada sobre las zonas geográficas donde se producen cada uno de estos trabajos de graduación y tesis, observamos que la gran mayoría son producidos al interior del Área Metropolitana de San Salvador (AMSS) con un total de 30. Dicho dato no es de extrañar, dado que la mayor parte de las 26 universidades se concentra en esta región, 17 universidades privadas y el mayor campus de la Universidad de El Salvador, única universidad pública del país (El Salvador, 2014). No obstante, el hecho que 9 producciones sean elaboradas fuera del AMSS, marca una pauta de que existe un interés sobre estas temáticas fuera de San Salvador. Al mismo tiempo, algunas de las producciones académicas que son radicadas en el AMSS, pero sus trabajos de campo fueron realizados en otras zonas del país como el caso de Díaz y Vega (2007) y Cañas y Escolera (2013).

Respecto al sector, privado o público, de las producciones académicas, la Universidad de El Salvador cuenta con la mayoría de ellas, un total de 20. Las restantes 19 se dividen entre la Universidad Dr. José Matías Delgado (5), Universidad Centroamericana José Simeón Cañas (5), Universidad de Oriente (2), Universidad Modular Abierta (2), Universi-

1378 Educação \& Realidade, Porto Alegre, v. 42, n. 4, p. 1375-1397, oct./dic. 2017. 
dad Francisco Gavidia (2), Escuela de Comunicación Mónica Herrera (1), Universidad Evangélica de El Salvador (1), Universidad Pedagógica de El Salvador (1). Esta situación es interesante, si se compara con Costa Rica, país del área Centroamericana, en el cual de las 54 producciones universitarias que se tiene conocimiento fueron producidas entre 19802013, 50 de ellas son producciones de universidades públicas y 4 de universidades privadas (Jiménez, 2014).

Después de haber realizado la revisión de cada una de las producciones académicas, comenzaron a surgir ejes temáticos, en los cuales se pueden agrupar separadamente las 39 producciones académicas encontradas. Estos ejes temáticos se han construido por medio de la comparación entre las producciones revisadas. Revelando que estas no han respondido a ninguna línea de investigación previa, sino más bien cada uno de los trabajos de grado y tesis de maestría surgieron para dar respuestas a fenómenos concretos o para discutir temáticas, como el acceso matrimonio por parte de personas del mismo sexo, que se tornó un tema mediático en fechas determinadas de los años 2005, 2006, 2009 y 2012.

La existencia de estas 39 producciones, lo considero como un éxito. En la academia salvadoreña aún se encuentran resistencias para entablar diálogos sobre temas de orientación sexual, identidad y expresión de género, dados los prejuicios existentes en la sociedad. Muestra de este hecho es que un $62 \%$ de la población salvadoreña no acepta la homosexualidad (Pew Research Center, 2013). Lo preocupante de este estigma (Goffman, 1988) es la producción de diversas formas de violencias, por ejemplo: el $85 \%$ de la población salvadoreña, consideran que otras personas tienen derecho de agredir a una persona trans/travestí por su identidad o su forma de ser (Asociación Comunicando y Capacitando a Mujeres Trans con VIH en El Salvador, 2013). Estos prejuicios en la mayoría de veces son extrapolados a las universidades.

Utilizando las palabras del antropólogo lingüista Rafael Lara-Martínez (2012) tanto el cuerpo y la sexualidad son borrados de los discursos públicos y oficiales, como resultado obtenemos que son remitidos al silencio y al tabú. En los ámbitos académicos aun la diversidad sexual no alcanza un estatus de respetabilidad académica. Por omisión académica se reproduce la jerarquía sexual que Gayle Rubin manifestó en la década de 1980, con la existencia de un Sexo "bueno" y un Sexo "malo" (Rubin, 1989, p. 140). En este caso, podemos extrapolar y asegurar que al interior de la academia salvadoreña existe una dualidad entre una buena investigación y una mala investigación. En la mala investigación estarían concentrados investigaciones que salieran fuera del parámetro heteronormativo binario, las cuales carecerían de respetabilidad o solidez académica para ser un área de estudio adecuada, respetable y pertinente para realizar las finalidades de la educación superior salvadoreña de investigación, docencia y proyección social.

No obstante, a todo ese escenario ideológico adverso, investigaciones sobre diversidad sexual fueron realizadas, las cuales proponen caminos para comenzar a reflexionar de forma amplia las manifesta- 
ciones diversas de la sexualidad. Para presentar cada uno de estas producciones se hace por medio de 4 ejes-binomios. Estos representan posiblemente los cuatro campos de tensiones que la sociedad salvadoreña posee en relación a los fenómenos y las temáticas que se relacionan a la Diversidad Sexual. Al interior de algunos de los ejes-binomios la información se agrupa por medio de bloques de producciones académicas que poseen algún tipo de relación, afinidades temáticas, semejanzas, aproximaciones teóricas o metodológicas entre sí.

\section{Prácticas Sexuales/Cuerpos}

Este eje está integrado por tres bloques. El primero versa sobre homosexualidad y VIH, el segundo hace referencia sobre prácticas sexuales y el tercer bloque habla sobre modificaciones corporales.

El primer bloque de este binomio-eje, al mismo tiempo es un trabajo pionero emprendido para el estudio de la diversidad sexual en El Salvador, también se observa la manutención de estereotipos que relacionan la homosexualidad y el VIH. Villatoro y Quintero (1998), siendo la primera incursión académica que trató temáticas sobre diversidad sexual indagaron sobre la práctica homosexual de la población masculina en la ciudad de San Salvador y su relación con el Sida. Las prácticas sexuales que ejercían estos cuerpos se relacionan a una enfermedad: VIH. Esta relación no es de extrañarnos para la época de la década de 1980, en donde existían más dudas que certezas sobre el VIH, sus formas de transmisión y las poblaciones que afectaba. Para el caso de Costa Rica, en la década de 1980 también se presenta el fenómeno VIH/SIDA como un eje principal en el abordaje académico, pero al mismo tiempo se aborda el activismo LGBT como segundo eje (Jiménez, 2014), área que no se ha discutido hasta el momento en El Salvador.

Pasados 25 años de la primera incursión académica que versó sobre Sida y homosexualidad, aunque la relación del binomio anterior no es cuestionada, los abordajes a las poblaciones sufren cambios. Por ejemplo, de prácticas homosexuales en la población masculina en 1988 se da paso a Poblaciones En Más Alto Riesgo (PEMAR) abordado por Cañas y Escolera (2013). Este cambio conceptual marca una mirada diferente hacia la diversidad sexual: visibilizando y diferenciando a las mujeres trans de los hombres gays y bisexuales, pero al mismo tiempo invisibilizando a las lesbianas y a los hombres trans por considerarlos de menor riesgos que las otras poblaciones. No obstante, pese a todos los procesos de sensibilización, el Acuerdo no 202 del Ministerio de Salud (2009), los lineamientos técnicos para el abordaje en salud de las personas LGBT (El Salvador, 2012), y la agencia de grupos, asociaciones, colectivos y organizaciones no gubernamentales (ONG) la homofobia, lesbofobia y transfobia aún persisten en el personal de salud, lo cual genera rechazos y acciones discriminatorias a personas de diversidad sexual que procuran ejercer su derecho a la Salud (Cañas; Escolera, 2013).

La ideología que pretende ser hegemónica sobre la relación VIH-Homosexualidad, también es aprovechada por las propias perso-

1380 Educação \& Realidade, Porto Alegre, v. 42, n. 4, p. 1375-1397, oct./dic. 2017. 
nas homosexuales y gay que proporciona un medio de trabajo y al mismo tiempo contribuye para cuestionar esos prejuicios existentes. Tal situación lo muestran Bendek y Ulloa (2015) al analizar la utilización de las redes sociales por parte de la Asociación Entre Amigos ${ }^{1}$ y su uso en la prevención del VIH. Esta producción universitaria tiene una característica especial, ya que desde su diseño, ejecución y resultado final tuvo el formato de consultoría, que proporcionó a la Asociación Entre Amigos como producto final una Propuesta de Plan Digital que abarca todas las áreas digitales de la Asociación, desde la atención al cliente, hasta procedimientos en crisis pasando por la gestión de contenido institucional (Bendek; Ulloa, 2015).

El segundo bloque, si bien es cierto se aleja formalmente de la relación VIH-Homosexualidad, esta se mantiene latente al adentrarse en los placeres, experiencias y prácticas sexuales, temáticas que en la academia poco se ha producido. Como afirma Lara-Martínez (2012, p. 241), "Sea desdén o tabú el silencio da cuenta de esas omisiones en las cuales nada se cultiva"; o el olvido de lo poco producido en los anaqueles de las bibliotecas de las universidades.

López, Martínez y Umaña (2012) nos presentan el primer trabajo sobre placeres, hasta cierto punto proscritos, entre mujeres adolescentes. Esta investigación procuró saber sobre la existencia o no de vivencias homosexuales entre mujeres adolescentes de 15 a 19 y la existencia de discriminación por tales prácticas al interior de un Centro Infantil de Protección Inmediata (CIPI) ${ }^{2}$. Entre sus conclusiones destacan que a las adolescentes internas en el CIPI "[...] genera ciertas condiciones negativas, entre ellas actos de discriminación, hacia algunas adolescentes que han experimentado vivencias homosexuales" (López; Martínez; Umaña, 2012, p. 1).

Por su parte, Baires, Pérez y Umaña (2014) exploran en las redes sociales su incidencia para que hombres accedan al trabajo sexual masculino. Sus indagaciones intentan aproximarnos a los lugares físicos de trabajo sexual masculino en San Salvador, el tipo de clientela y la relación servicio/cliente homosexual que se establece. Entre sus hallazgos destacan que la relación servicio/cliente homosexual puede ser solamente la contratación como un acompañante social, más que la ejecución de un servicio sexual.

Modificar el cuerpo conforme a una identidad de género es lo que se explora en este tercer bloque. La identidad del transgénero se hace presente, en este caso definida como "[...] la persona que vive bajo otro género, pero que no buscan una cirugía de reasignación de sexo como el transexual o no tiene acceso a ella; y prefieren solo algunos tratamientos como la hormonización" (Cabrera; Parada, 2009, p. 4). En este bloque surgen muchas preguntas para ser contestadas por la institucionalidad, al saber que las prácticas de feminización de mujeres trans no cuentan con asistencia médica para realizarlas, lo cual coloca en riesgos para la salud al someterse a procesos hormonizadores sin un acompañamiento médico (Cabrera; Parada, 2009). En esta oportunidad solamente se habla de los procesos de transición de género de lo masculino para lo 
femenino, pero no lo contrario de lo femenino a lo masculino, ya que la trans masculinidad en 2009 no era nombrada en El Salvador, sino hasta 2014 que el primer colectivo de hombres trans surge y coloca sus demandas junto con las otras identidades LGBTI.

\section{Matrimonio/Familias}

Este eje-binomio es dominado exclusivamente por las Ciencias Jurídicas, las cuales han tratado de analizar los diferentes matices jurídicos para que personas del mismo sexo posean el acceso a la institución civil del matrimonio. Por ser la misma temática vista desde puntos diferentes de las Ciencias Jurídicas este eje-binomio no está dividido en bloques.

En el año 2005 comenzó un proceso de reforma constitucional para prohibir el acceso al matrimonio a parejas del mismo sexo, proceso que aún se encuentra vigente. Tres años antes que todo este proceso convulso iniciara Hernández y Ventura (2002) realizando un análisis jurídico-doctrinario de los derechos constitucionales aplicables a parejas homosexuales, en este caso de la ciudad de San Miguel demostraron que la garantía constitucional de no discriminación no se cumple cuando se trata de parejas de personas del mismo sexo, explicitando que existe una negación del derecho a la seguridad jurídica.

Ascencio, Calderón y Sevilla (2008), realizan un análisis jurídico de las propuesta de reformas constitucional para prohibir el acceso al matrimonio y la adopción por parte de parejas del mismo sexo. En forma general, más que analizar los fundamentos jurídicos que sustenten esa reforma, discuten como la homofobia adquiere diferentes formas en los ámbitos sociales e institucionales. Continuando con esta temática, Martínez y Reyes (2008) discuten los conflictos posibles entre diversos tratados firmados por El Salvador, si estos llegaran a ser activados para reconocer los matrimonios de salvadoreños del mismo sexo efectuados en otros países que reconocen este derecho.

En Morán, Palma, Torres y Zelaya (2011) y De León, Melgar y Velásquez (2011) se muestran claramente dos puntos opuestos al discutir el acceso al matrimonio desde el área jurídica. Morán, Palma, Torres y Zelaya (2011) reproducen el discurso fundamentalista religioso y conservador en el ámbito académico, y se valen de la moralidad religiosa como excusa para negar por cualquier medio derechos constitucionales a parejas del mismo sexo. En el otro extremo, De León, Melgar y Velásquez (2011), concluyen que el no reconocimiento de los matrimonios a parejas del mismo sexo, tanto el Estado como la sociedad incurren en una violación a los Derechos Humanos.

En Andaluz, Guzmán y Pineda (2012), lo que cabe destacar de esta producción es la forma como disfrazan la discriminación y homofobia por parte de diversos sectores de la sociedad por medio de discursos que llaman hacia la tolerancia, pero que al mismo tiempo niegan categóricamente derechos a las personas LGBTI. En cuanto Marroquín (2012) a través de un estudio jurídico comparado entre México, España 
y Argentina muestra que en El Salvador se puede permitir el acceso al matrimonio a personas del mismo sexo ya sea por la vía legislativa o por medio de recursos de amparo ante la Corte Suprema de Justicia.

Para finalizar este eje-binomio, Bernal, Carballo y Ventura (2015) realizan una apología del discurso conservador y fundamentalismo religioso, desde una visión jurídica, para sustentar la prohibición del matrimonio entre personas del mismo sexo por medio de la necesidad de protección a la niñez. Argumentando que la homosexualidad es antinatural, conducta desviada y comportamiento inmoral. Por lo cual, sí se permitiera el matrimonio entre personas del mismo sexo sería legalizar y promover supuestas características negativas en niños, niñas, adolescentes y jóvenes (Bernal; Carballo; Ventura, 2015).

\section{Identidades/Prejuicios}

Este eje-binomio se presenta una paradoja. La construcción de identidades sociales como gay, lesbiana o transexual conlleva al mismo tiempo una visibilización y la manifestación de prejuicios. Prejuicios hacia personas de identidades LGBTI no es algo nuevo, pero si es algo que se oculta. Al momento que estos dan muestra de su existencia, los prejuicios comienzan adquirir formas de discriminaciones, en este caso como homofobia social e institucional. Este eje cuenta con dos bloques, el primero referido a identidades LGBTI y el segundo narra algunas de las formas que adquiere la homofobia social e institucional en El Salvador.

En este bloque conoceremos producciones universitarias que habla sobre alguna identidad LGBTI. Hernández y Hernández (1998) inician hablándonos de las minorías homosexuales. Por su parte Cruz, Sánchez y Azcuñaga (1999) presentan a los travestidos en su investigación periodística. Travestido es un concepto discriminador que hasta la fecha se utiliza para nombrar a las mujeres trans. Garay, Salguero y Segovia (2002) al inicio del presente milenio hablan sobre las mujeres lesbianas, por medio de una caracterización psicológica utilizando el test de la Figura Humano Karen Machover, el inventario de autoevaluación precisa y entrevistas por áreas de desarrollo evolutivo, social, salud, identidad, sexualidad y afectiva. Chávez y Morales (2002) mencionan a los educandos con tendencia homosexual. A parte de dar cuenta sobre la relación familiar de los homosexuales que revelaron su orientación sexual. López y Naves (2006), dan cuenta de la denominación como grupo minoritario a los homosexuales y colateralmente describen y mencionan sus espacios de sociabilidad al describir su trabajo de campo. En el trabajo de grado y documental social ${ }^{3}$ Historia de Vida de una Mujer Transexual (Saca; Vázquez; Álvarez, 2006), se registra una nueva identidad: mujer transexual. Ríos (2009) documenta y analiza el surgimiento de identidades políticas, como el caso de las mujeres lesbianas feministas, al realizar un estudio de caso que tenía como protagonista a Fátima Polanco integrante del Colectivo Las Desclosetadas.

Educação \& Realidade, Porto Alegre, v. 42, n. 4, p. 1375-1397, oct./dic. 2017. 
En este segundo bloque se presentan producciones que aborden temáticas relacionadas a las diferentes formas que adquiere la homofobia. Hernández y Hernández (1998) inician este recorrido desde el machismo como expresión cultural dominante en el país. El machismo genera diferentes formas de discriminación. Una de estas se presenta en el área del trabajo, además por su connotación de identidad nacional es de extrema importancia. Así tenemos que las personas homosexuales se enfrentan a una desigualdad laboral (Hernández; Hernández, 1998), la cual conlleva si además de su orientación sexual la persona manifiesta una identidad de género fuera del patrón binario sexual, a una discriminación laboral que obliga a muchas mujeres trans a optar por dos caminos laborales: la prostitución o el trabajo en salones de belleza.

Cruz, Sánchez y Azcunaga (1999), indagan sobre el rechazo de los homosexuales en el ámbito laboral, en este caso, en los empleos formales, al analizar la política de contratación laboral de diversas empresas. Girón, Toledo y Torres (2007) presentan un estudio que coloca en discusión el ámbito laboral, la orientación sexual, la expresión de género y la identidad sexual en personas del sexo masculino entre 16 a 30 años de edad que laboran en salones de belleza de la ciudad de Santa Ana. Por su parte Alvarado, Klüver y Reyes (2013) abordan las diferentes manifestaciones de la discriminación laboral que Stacy y Cristina, mujeres trans padecen al interior de la sociedad salvadoreña marcada por el machismo. Garay, Salguero y Segovia (2002) y Mejía, Ortiz y Roca (2013) manifiestan que la discriminación laboral en las mujeres lesbianas puede ser disminuida con procesos de adaptación que rozan el ocultamiento de sus identidades en el ámbito laboral. Para finalizar, Realegeño y Valldeperas (2007) exponen que la homofobia, aparte de violencias físicas y muertes, también incide a nivel de la salud mental de las personas.

Alfaro y Cortez (2012) muestran en el corto Tenemos que hablar ${ }^{4}$, que la homofobia también se hace presente al interior de las relaciones familiares al momento de descubrir o manifestar, uno de sus integrantes una orientación sexual o identidad de género diferente a la heterosexual. Rosales, Sánchez y Segovia (2011) muestran que la filiación religiosa de la familia incide en la percepción negativa a la homosexualidad. No obstante, tanto López y Naves (2006) y Abrego (2013) dan cuenta de los procesos de aceptación familiar, con sus dificultades, de la orientación sexual de uno de sus miembros.

Existen producciones académicas que tratan sobre la descripción de la homofobia, y al mismo tiempo existen otros trabajos que estudian este fenómeno. Guzmán y Merlos (2006) no presentan mayores contribuciones, aparte de describir el fenómeno, no cuestionando sus fundamentos sociales, religiosos, culturales, educativos. Por otra parte, existe trabajos pioneros que tratan de colocar en una discusión académica sería el fenómeno de la homofobia y sus forma de ocultamiento: el discurso correctamente político y la tolerancia como el caso de Gómez, Marcenaro y Meza (2013). Relacionado a lo anterior, Giró y Martínez (2012) desde un estudio cuantitativo exploran la relación de dogmatismo y homofobia en jóvenes universitarios del AMSS. Como resultado se obtu- 
vo que existe una relación entre el nivel de dogmatismo y la utilización moderada de formas indirectas de homofobia que se manifiestan como incomodidad, inseguridad, disgusto o miedo. García, Najarro y Ramos (2014) presentan una investigación sobre recursos cognitivos para comprender y valorar la homosexualidad por parte de jóvenes universitarios del AMSS. Para finalizar Contreras y López (2014) exponen que existe mayor aceptación social de la homosexualidad en los estudiantes del campus central de la Universidad de El Salvador.

En el caso de Parada, Escobar y Torres (2008) ${ }^{5}$ aunque en su título se manifiesta que analizaría la discriminación social hacia personas con tendencias homosexual, lo más sobresaliente y paradigmático de esta producción universitaria es el lugar de realización del trabajo de campo: Grupo de Rehabilitación Homosexual Cambiando Rumbo. Cualquier postulado ético contra los procesos de reconversión o reeducación sexual para curar la homosexualidad son olvidados. No se cuestiona en ningún momento el proceso de terapia correctiva que se presenta como formación espiritual en este grupo.

\section{Derechos/Ciudadanías}

Este eje-binomio se fundamenta en los procesos y la búsqueda de reconocimiento social por parte de las poblaciones LGBT, aunque incipientes, como sujetos de derechos en igualdad de condiciones a las demás personas. La visión jurídica nuevamente domina este eje de análisis, por tal motivo no existe una división en bloques.

La primera producción académica que versa sobre este proceso de reconocimiento es la discusión que presenta Aguilera, Avilés y Magaña (2005), sobre sí se debe o no aplicarse la Ley de Violencia Intrafamiliar vigente en El Salvador cuando sucede al interior de una pareja de personas del mismo sexo un acto de violencia. Esta discusión, por sorprendente que parezca, surge desde un caso registrado en el Juzgado de Familia de la ciudad de Santa Ana. Este fue resuelto reconociendo la existencia de violencia intrafamiliar entre una pareja de hombres y se aplicó la normativa jurídica para este caso sin ningún proceso de diferenciación o discriminación por orientación sexual o identidad de género.

Este reconocimiento de Derechos, también debe de darse incluso cuando el derecho a la libertad está restringido en personas homosexuales. Como vimos en el eje anterior la existencia de la homofobia se manifestaba en los ámbitos laborales y familiares en forma de discriminación, en el caso de personas homosexuales cuando son privados de libertad, la homofobia se puede presentar de forma violenta como lo presentan Díaz y Vega (2007, p. 103), ya que las personas LGBTI son o pueden ser “[...] objeto de violación y agresión física y sexual por parte de custodios e internos del centro penal [...] siendo vulnerados muchos de sus Derechos Humanos por el simple hecho de ser homosexuales".

Por último, Ochoa y López (2015) reconocen la existencia de avances en materia de Derechos Humanos, como ha sido la emisión 
del Decreto Ejecutivo no 56 que prohíbe las discriminación motivadas por orientación sexual en la administración pública, la creación de la Dirección de Diversidad Sexual y la Mesa Permanente sobre Derechos Humanos de la población LGBTI,

\begin{abstract}
A pesar que existen avances en el reconocimiento de los derechos humanos de la población LGBTI en El Salvador, su situación actual sigue siendo la de vulnerabilidad a la discriminación y violencia, porque hace falta una regulación jurídica que responda a lo preceptuado por la Constitución de la República e instrumentos internacionales en materia de derechos humanos (Ochoa; López, 2015, p. 180).
\end{abstract}

La construcción de ciudadanías y el ejercicio pleno de los Derechos Humanos de personas salvadoreñas LGBT, considero que será una nueva frontera a ser conquistada por la sociedad salvadoreña en su conjunto.

\title{
La Propuesta: qué mirar desde los estudios sobre diversidad sexual
}

Las producciones académicas universitarias que fueron construyendo ejes de investigación de la diversidad sexual, fue un proceso espontaneo y hasta cierto punto no planificado. Teniendo en cuenta esta situación, se debe articular en los ejes construidos otras miradas para analizar la diversidad sexual. Así que daré paso a mencionar otras áreas para reforzar los ejes ya constituidos.

\section{Prácticas sexuales/Cuerpos}

El armario académico adquiere en 1988 una mirada negativa con el estudio relacionado del binomio Homosexualidad-VIH, y considero que esa relación hasta el momento no se ha podido desasociar en la academia salvadoreña. En tal sentido, considero que las prácticas sexuales de la diversidad sexual, deben de verse ya no desde el sentido negativo que el pensamiento conservador le ha imprimido, haciendo una relación casi inquebrantable de VIH-Homosexualidad. En los Estudios sobre Diversidad Sexual se debe de analizar la sexualidad desde un sentido más amplio que trate de abarcar el abanico multifacético que este aspecto de la vida humana envuelve. Así el estudio de la sexualidad puede realizarse desde el placer hasta llegar al ejercicio del poder y la construcción de jerarquías entre las personas que establecen vínculos sexuales entre ellas. Sin olvidar prácticas sexuales que están en aumento en este momento como los clubes de masturbación, relaciones sadomasoquistas, exhibicionismos, entre otras.

Aunque invisibles, vulnerables y discriminados la comunidad de diversidad sexual ha ido creando espacios de sociabilidad para escapar aunque sea por unas horas del estigma y ser simplemente seres humanos que desean divertirse. Los espacios de sociabilidad pueden y deben investigarse en diversos sentidos, desde guetos en una sociedad conser- 
vadora, espacios sociales de ruptura, espacios de encuentro afectivos o hasta lugares de construcción de identidades políticas LGBTI.

La manipulación del cuerpo y sus procesos para conectarlo con la identidad de género que se posee es una área de investigación que se debe de profundizar. Así los procesos de hormonización o de reasignación sexual tanto femenino como masculino deben de ser foco de atención de las ciencias médicas y sociales. Es necesario investigar sobre las políticas públicas y el ejercicio al derecho a la salud de poblaciones trans, con el intuito de disminuir sus sufrimientos de manifestar una identidad sexual y la imposibilidad de modificar su cuerpo sin riesgos para su salud.

La heterosexualidad al ser la norma común es al mismo tiempo una temática invisible para la academia, por tal razón su estudio y respectivo análisis debe de iniciar. En ese mismo sentido el estudio y análisis de la bisexualidad tanto en hombres como mujeres, es una temática que hasta el momento no ha sido abordada, por lo cual merece atención en la producción académica.

La intersexualidad, se puede considerar como una incógnita en la producción científica de las universidades salvadoreñas, y por tal motivo un área de estudio urgente para ser abordada.

\section{Matrimonio/Familias}

En este eje, considero necesario que se abra a otras disciplinas, ya que la única que ha emitido sus opiniones son las Ciencias Jurídicas. En tal sentido otras disciplinas como la Ciencia Política, Sociología, Antropología, Historia, Psicología entre otras deben de realizar sus aportes en la discusión del hecho social de parejas del mismo sexo en su lucha por el reconocimiento del derecho civil de contraer matrimonio.

Es un hecho que existen ya parejas del mismo sexo en El Salvador y también es un hecho que estas parejas en muchos casos están a cargo de hijos e hijas, constituyendo familias, por tanto el estudio de las homoparentalidades es un área que no se puede dejar de obviar en este eje-binomio.

Los abordajes de la homosexualidad al interior de las familias, siempre se supone una horizontalidad de aceptación de los padres hacia los hijos; pero también existen otras posibilidades no abordadas: cuando los padres revelan para sus hijos su homosexualidad. En este mismo sentido, pueden existir abuelos que revelan a sus familias su homosexualidad. Estas temáticas no deben de perderse de vista para su análisis al estudiar familias diversas.

\section{Identidades/Prejuicios}

El campo de construcción de identidades es uno de los más amplios y poco explorados con rigurosidad en El Salvador. En las diferentes producciones académicas, para colocar un ejemplo, se ha percibido que 
la identidad homosexual tiene un tránsito desde ser considerada como una práctica sexual (Villatoro; Quintero, 1988), pasando por minoría social (Hernández; Hernández, 1998), tendencia (Chávez; Morales, 2002) personas homosexuales (Abrego, 2013), hasta población de riesgo (Cañas; Escolero, 2013) y sujeto de Derechos (Ochoa; López, 2015). Estas variables y modificaciones requieren mayor análisis. Así desde de una perspectiva de la genealogía que propone Foucault (1993) sería interesante estudiar la conformación de las identidades LGBTI en El Salvador.

La investigación sobre diversidad sexual debe tomar en cuenta los marcadores sociales de la diferencia; entre los cuales puedo mencionar el de generación, geografía, género, clase social, etnia y raza. Así cuando se habla de generación podemos colocar la mirada en cómo los jóvenes LGBTI están construyendo sus identidades, sus tensiones, dificultades o facilidades. Al mismo tiempo no podemos olvidar tanto a personas adultas como a los adultos mayores, para estos sectores es necesario conocer el cómo fueron construyendo sus identidades y cómo la resignifican en esta época que se diferencia a las décadas anteriores.

En el marcador social de geografía, los procesos de investigación pueden explorar la forma de construcción de identidades en las áreas rurales, las urbanas, por zonas geográficas, por departamentos e incluso por municipios, mostrando con este proceso las diferencias que existen entre territorios próximos. Tomando en cuenta la alta cantidad de salvadoreños fuera del país y en proceso, por medio de vínculos de investigación entre universidades, se debería de investigar sobre personas salvadoreñas LGBTI en contextos migratorios, de tránsito y de llegada a sus destinos.

En cuando al género, se debe observar las formas de construcción de identidades diferenciadas entre hombres y mujeres, y no olvidando el estudio de los procesos de construcción de lo femenino y lo masculino en las personas trans, temática inédita hasta el momento. Sería interesante conocer las articulaciones y tensiones políticas en la utilización del concepto género entre mujeres lesbianas, lesbofeministas y el movimiento de mujeres ampliado. Relacionado a lo anterior, la inclusión o no de las mujeres trans como parte del movimiento feminista también puede ser una discusión estimulante.

La clase social como marcador de la diferencia no se debe de remitir exclusivamente a las personas con bajos ingresos económicos las cuales son catalogadas como pobres; sino más bien, realizar procesos de investigación en las adaptaciones, tensiones y resignificaciones de identidades LGBTI en las élites económicas y entre los trabajadores profesionales, concepto que utilizo para designar a la clase media en El Salvador. En este mismo sentido de clase social, una temática apremiante sería estudiar la existencia de personas salvadoreñas LGBTI y la construcción de sus identidades al interior de las maras $^{6}$, una temática no explorada por la complejidad del campo y su peligrosidad, pero al mismo tiempo un tema tentador, retador y provocante.

Otra temática de interés es la construcción de identidades políticas. Esta temática se conecta con la historia del movimiento organizado

1388 Educação \& Realidade, Porto Alegre, v. 42, n. 4, p. 1375-1397, oct./dic. 2017. 
de diversidad sexual al interior del país. Esta investigación debe interaccionar los marcadores sociales de la diferencia vistos anteriormente, pero desde una perspectiva interseccional (Piscitelli, 2008). La interseccionalidad permitiría conocer con mayor profundidad la dinámica de las identidades políticas, sus tensiones, encuentros y desencuentros en ese proceso de construcción, estabilización y resignificación de las mismas. Las lesbianas feministas son una de las identidades políticas interesantes de investigación por ejemplo, o en un futuro no muy lejano, las mujeres y hombres trans feministas serán identidades políticas de interés para su estudio.

Aunque en este momento aún se encuentran en proceso de construcción y estabilización las identidades LGBTI, es necesario introducir la teoría queer en los debates académicos y políticos. El introducir esta discusión es necesaria desde el punto de vista que contribuiría para cuestionar la consolidación de discursos hegemónicos como lo heteronormativo o lo androcéntrico que reproducen procesos de discriminación, homofobia y machismo al interior de los propios colectivos y grupos de diversidad sexual.

La propia historia de la homosexualidad en El Salvador es un área que está a la espera que se comiencen procesos de investigación. La influencia de los medios de comunicación y la construcción de identidades de diversidad sexual, es un campo no explorado y sería interesante indagar en esta área. De la misma forma las manifestaciones de diversidad sexual al interior de las fiestas patronales y los discursos políticos que se crean al interior de estas, es una temática no explorada hasta el momento. Las diversas organizaciones LGBT desde 1997 promueven actos de visibilidad política, los cuales desde el año 2009 han aumentado, sería muy interesante el estudio de estos actos y su impacto o no en la modificación de patrones de discriminación por orientación sexual, identidad y expresión de género.

Por último, ¿en qué medida la construcción de identidades sociales y políticas de diversidad sexual pueden contribuir en la disminución de procesos de discriminación hacia personas salvadoreñas LGBTI? Es una pregunta que queda abierta, ya que una respuesta definitiva no se posee aún.

\section{Derechos/Ciudadanías}

La construcción de ciudadanías y el ejercicio pleno de los Derechos Humanos de personas salvadoreñas LGBTI, pasa inexorablemente por el ejercicio pleno de los derechos individuales y sociales estipulados en la Constitución Política de El Salvador. En este sentido el derecho a la Educación, Salud, Cultura, Trabajo, Previdencia social, matrimonio, adopción, pero sobre todo el principio de Igualdad deben de ejecutarse reconociendo la existencia de la diversidad sexual.

Una temática de interés en indagar en este eje es el cómo opera el conservadurismo religioso en la arena política que se opone al ejercicio pleno de derechos de las personas de diversidad sexual, utilizando discursos políticamente correctos como el de la tolerancia, pero que al

Educação \& Realidade, Porto Alegre, v. 42, n. 4, p. 1375-1397, oct./dic. 2017.1389 
¿El armario está abierto?

final este no permite el reconocimiento de derechos plenos y se crea una concepción de que el amor al prójimo que tantas veces se predica en las iglesias de matriz católica-protestantes, queda condicionado a que el prójimo sea presumiblemente heterosexual como el que emite el discurso para ser beneficiado por los derechos. No obstante, si el prójimo es diferente, este no es merecedor de ser un sujeto de derecho, y en vez de la promoción de derechos, lo que se procura es la supresión de estos, recurriendo a discursos moralistas para justificar esa tolerancia a la diversidad sexual, que al mismo tiempo niega su pleno reconocimiento como sujetos de derechos.

Se debe de emprender una investigación interdisciplinar e interseccional para analizar y comprender el cómo la violencia que se ejecuta contra personas de diversidad sexual es un mecanismo de control que impide su constitución como sujetos de derechos plenos. Como resultado de estos procesos de violencia, muchas personas salvadoreñas LGBTI se ven forzados a realizar procesos migratorios que en más de un caso concluyen en muerte. No obstante, este fenómeno social no ha sido estudiado a profundidad como se mencionó anteriormente.

Para finalizar, aunque son incipientes, existen en El Salvador algunas políticas públicas en ejecución: Dirección de Diversidad Sexual, lineamientos técnicos para el abordaje en salud de las personas LGBT, el Decreto Ejecutivo no 56, el Acuerdo del Ministerio de Salud no 202 y la Mesa Permanente sobre Derechos Humanos de la población LGBTI, lo cual debe de ser investigado para conocer el impacto de estas políticas públicas en el reconocimiento de ciudadanía de las personas de diversidad sexual.

\section{Los Retos: educación superior universitaria y diversidad sexual}

El primer y mayor reto de todos posiblemente es la deconstrucción del tabú de la sexualidad en El Salvador en las diferentes áreas de la vida, incluyendo en la academia. La sexualidad esta confinada al campo de los instintos y lo privado, lo cual ha fomentado el silencio sobre esta área de la vida individual y social. El silencio construye el armario del tabú para esconder las relaciones de poder y jerarquías que son ejercidas desde la sexualidad, la cual es considerada aceptable únicamente aquella que sea parte del sistema binario reproductivo y heterosexual. La educación superior representada por las diferentes universidades no escapa a la reproducción de este sistema, el cual debe de comenzar a cuestionar.

Concatenado a lo anterior, el segundo reto sería, la construcción de una respetabilidad académica a la investigación de la sexualidad. Respetabilidad en el sentido de ser considerada como contenido de docencia, área de estudio y campo de investigación; colocando así lo privado como una categoría política de extrema importancia que estructura nuestra sociedad, en la cual se establecen jerarquías de acuerdo al ejercicio de la sexualidad que ejecutan los individuos.

1390 Educação \& Realidade, Porto Alegre, v. 42, n. 4, p. 1375-1397, oct./dic. 2017. 
En tercer lugar, la manutención del principio Laico en las universidades no confesionales es un reto. Por una parte en las universidades privadas, según los lineamientos de su junta directiva, que en muchas ocasiones son los principales accionista de la universidad, sino sus dueños; emiten políticas internas fundamentadas en preceptos religiosos conservadores, los cuales menoscaban los procesos de investigación sobre temáticas de diversidad sexual. Ahora bien en el ámbito público, el embate de sectores fundamentalistas religiosos puede coaptar el desenvolvimiento de procesos de investigación, de acciones de proyección social y de docencia sobre temas de diversidad sexual. Conectado a lo anterior, la introducción de temáticas de diversidad sexual al interior de universidades que se denominan como confesionales, también es otro reto.

Un desafío de primer orden es la institucionalización de procesos de investigación sobre diversidad sexual por medio de centros, núcleos de estudio o líneas de investigación en posgrados. En primer lugar, sería necesario que grupos de interés se movilicen para la creación de estos espacios académicos. En segundo punto es el entablar diálogos con los administradores institucionales para que acepten la importancia del funcionamiento de este tipo de centros y los beneficios que pueden aportar a la sociedad. Ligado a lo anterior, el financiamiento de estos centros o núcleos de investigación se transforma en un punto base para iniciar actividades de investigación sobre temáticas de diversidad sexual. En el caso de la Universidad de El Salvador, la procura de fuentes de financiamiento es más complicado, dado el aislamiento académico que heredó del tiempo de la guerra y que hasta el momento no ha logrado superar (Bonilla, 2013).

Uno de los mayores retos, no únicamente de los estudios de diversidad sexual en El Salvador, sino de toda la educación superior es la aplicación de la interdisciplinariedad. La concepción de disciplinas separadas y que no se pueden aproximar para estudiar los fenómenos sociales es una tradición académica de fuerte raigambre que presenta resistencias para ser modificadas. En este mismo orden, la participación de las ciencias exactas o las tradicionalistas - y por qué no decirlo machistas también - ingenierías y arquitectura es un reto que se aproximen y participen desde sus campos de saberes en el estudio de la diversidad sexual.

A nivel teórico y práctico una de las discusiones que los estudios sobre Diversidad Sexual deben realizar son sus conexiones y tensiones entre el feminismo, las teorías de género y la teoría queer, y la forma particular de uso de los conceptos de estas teorías en el territorio salvadoreño.

Otro aspecto de importancia es lograr que lo acumulado de experiencias en la práctica de activistas de diversidad sexual pueda ser incorporado en los procesos académicos de los Estudios sobre Diversidad Sexual. Reconociendo que estos no pueden rechazar esa experiencia valiosa que en más de 20 años que el movimiento de diversidad sexual en el país ha acumulado. 


\section{Palabras de Cierre}

Al inicio del artículo se manifestó una provocación en forma de pregunta: ¿Existen Estudios sobre Diversidad Sexual en las universidades de El Salvador? Después del recorrido realizado se puede afirmar que han existido producciones académicas universitarias de trabajos de grado y maestría que su eje principal era discutir temáticas de diversidad sexual. No obstante, se debe de mencionar, que todo lo anterior no ha respondido ni a políticas ni a líneas de investigación específicas al interior de las universidades, por tanto no se pueden nombrar como Estudios sobre Diversidad Sexual, aunque se reconoce su existencia, pero al ser procesos esporádicos y no sistematizados sus miradas sobre Diversidad Sexual llega a ser someras, por lo cual no conforman un campo de estudios en estricto orden.

Las producciones académicas descritas han surgido por interés particulares, que tuvieron la intención de aproximarse a las realidades de personas salvadoreñas LGBTI. Pese a los contextos negativos de la sociedad y muchas veces reflejados al interior de la academia, abrieron los espacios para que estas discusiones comenzaran a adquirir un status de respetabilidad en el ámbito académico. No obstante, para que existan Estudios de Diversidad Sexual, estos deben de contar con una definición previa, y para ello es necesario contar con la estructuración de núcleos, centros o como mínimo con posgrados que posean líneas de investigación sobre temáticas de diversidad sexual.

La provocación que estimulo este análisis ha tenido una respuesta. En cuanto a la interrogante del título ¿está abierto el armario? se puede decir que aun los armarios de la academia salvadoreña están cerrados. Ahora bien, sólo queda preguntar para concluir: ¿Cómo podemos abrir los armarios de las universidades salvadoreñas? ${ }^{7}$

Recibido en 02 de febrero de 2016 Aprobado en 23 de abril de 2017

\section{Notas}

1 La Asociación Salvadoreña de Derechos Humanos Entre Amigos es la organización de diversidad sexual de mayor vida institucional en El Salvador, fue fundada en 1994.

2 Dependencia gubernametal cuya finalidad es la atención provisional, definitiva o detención administrativa (por resguardo) de niños/as y adolescentes que se encuentran en vulnerabilidad familiar, comunitaria o social de grave riesgo.

3 Para ver el documental, disponible en: <https://www.youtube.com/watch?$\mathrm{v}=$ MGfVU2hwHAo\&list=UUgnrpU_gsKHaZSoapgwPDFQ\&index $=10>$.Acceso en: 19 ene. 2016.

4 Para ver el corto, disponible en: <https://vimeo.com/54475605>. Acceso en: 19 ene. 2016.

5 Esta producción, a pesar que se encuentra catalogada como trabajo de grado en la Universidad Evangélica de El Salvador, su estructura es similar a la de un trabajo de cátedra.

1392 Educação \& Realidade, Porto Alegre, v. 42, n. 4, p. 1375-1397, oct./dic. 2017. 
6 Las Maras son grupos sociales urbanos o suburbanos marginales prioritariamente, de adolescentes y adultos jóvenes hombres mayoritariamente que controlan un territorio, con cierto nivel de acoso/acuerdo a/con los residentes locales y hostilidad hacia los externos, con una estructura organizacional, participación en actos de comercio legales e ilegales, algún grado de solidaridad interna basada en la identidad, restricciones y reglas propias, y la participación colectiva en diversas formas de violencia armada organizada (Carranza, 2005).

7 Agradecimiento del autor: Quiero dejar constancia de mi gratitud a Marcos Nascimento por las discusiones y sugerencias para la estructuración de los ejes-binomios. También quiero agradecer a Natalia Rivera, Rina de Aquino, Albert Girón, Josué Gómez, Andrés Orellana y Gabriel Gasteazoro por su ayuda para obtener copias físicas o virtuales de algunas de las producciones universitarias analizadas.

\section{Referencias}

ABREGO, Joselin. La Influencia de la Aceptación Familiar en Personas Homosexuales, Caso Atendido en la Clínica de la Universidad Dr. José Matías Delgado. 2013. 54 f. Monografía (Licenciatura en Psicología) - Facultad de Ciencias y Artes 'Francisco Gavidia', Escuela de Psicología, Universidad Dr. José Matías Delgado, Antiguo Cuscatlán, 2013.

AGUILERA, Norma; AVILÉS, Rosa; MAGAÑA, Iris. Principio de Igualdad como Garantía Constitucional en la Problemática de Parejas Homosexuales en los Casos que se Aplique la Ley contra la Violencia Intrafamiliar. 2005. 113 f. Monografía (Licenciatura em Ciencias Jurídicas) - Universidad de El Salvador, Santa Ana, 2005.

ALFARO, Johanna; CORTEZ, Jaime. La Homosexualidad, Símbolo de Aceptación e Igualdad Social. 2012. 86 f. Monografía (Licenciatura em Ciencias de la Comunicación) -Universidad Dr. José Matías Delgado, Antiguo Cuscatlán, 2012.

ALVARADO, Jorge; KLÜVER, Alejandro; VALENCIA, Juan. Monografía Especializada del Falso Documental: viviendo diferente. 2013. 42 f. Monografía (Licenciatura en Ciencias de la Comunicación) - Universidad Dr. José Matías Delgado, Antiguo Cuscatlán, 2013.

ANDALUZ, Norma; GUZMÁN, Rafael; PINEDA, Yeni. La Regulación Constitucional de la Prohibición de Matrimonio entre Personas del Mismo Sexo. 2012. 94 f. Monografía (Licenciatura en Ciencias Jurídicas) - Universidad Modular Abierta, San Salvador, 2012.

ASCENCIO, Emely; CALDERÓN, Lidia; SEVILLA, María. El Matrimonio Homosexual. 2008. 302 f. Monografía (Licenciatura en Ciencias Jurídicas) - Universidad de El Salvador, San Miguel, 2008.

ASOCIACIÓN COMUNICANDO Y CAPACITANDO A MUJERES TRANS CON VIH EN EL SALVADOR (Comcavis Trans). Informe para la Audiencia ante la Comisión Interamericana de Derechos Humanos sobre la Situación de Violencia Contra la Población de Mujeres Trans en El Salvador. San Salvador: Comcavis Trans. 2013.

BAIRES, Gabriela; PÉREZ, Irvin; UMAÑA, Karla. Las Redes Sociales como Medio de Acceso al Trabajo Sexual Masculino en El Salvador. 2014. 109 f. Monografía (Licenciatura en Ciencias de la Comunicación) - Universidad Dr. José Matías Delgado, Antiguo Cuscatlán, 2014. 
¿El armario está abierto?

BENDEK, Ana; ULLOA, Carlos. Análisis de la Web 2.0 de la Asociación Entre Amigos LGBTI de El Salvador y su Uso en la Prevención del VIH. 2015. 256 f. Monografía (Licenciatura en Ciencias de la Comunicación) - Universidad Dr. José Matías Delgado, Antiguo Cuscatlán, 2015.

BERNAL, Sindy; CARBALLO, Carlos; VENTURA, Lilian. La Protección de la Niñez en la Propuesta de Reforma a los Arts. 11 y 14 del Código de Familia, en la Prohibición del Matrimonio de Personas del Mismo Sexo así Nacidas como Mecanismos de Protección de la Familia. 2015. 272 f. Monografía (Licenciatura en Ciencias Jurídicas) - Universidad de El Salvador, San Miguel, 2015.

BONILLA, Adolfo. Breve Reflexión sobre la Reforma Académica en la Facultad de Ciencias y Humanidades. Revista Humanidades, San Salvador, v. 1, n. 1, p. 9-17, mayo/ago. 2013.

CABRERA, Juan; PARADA, Luz. Prácticas de Feminización y sus Manifestaciones en la Salud en Transgeneros de la Zona Metropolitana de San Salvador, Noviembre 2008-Febrero 2009. 2009. 89 f. Informe Final (Maestría en Servicios Integrales en Salud Sexual y Reproductiva) - Facultad de Medicina, Universidad de El Salvador, San Salvador, 2009.

CAÑAS, Ana; ESCOLERA, Sugey. Determinación del Grado de Aceptación del 'Programa VICITS' en Homosexuales, Unidad Comunitaria en Salud San Miguel, Diciembre/2012 - Enero/2013. 2013. 42 f. Monografía (Medicina) - Universidad de El Salvador, San Salvador, 2013.

CAREAGA, Gloria; CRUZ, Salvador. Sexualidades Diversas: aproximaciones para su análisis. México D. F.: Porrúa. 2004.

CARRANZA, Miguel. Detención o Muerte: hacia dónde van los niños 'pandilleros' de El Salvador. In: DOWDNEY, Luke (Org.). Ni Guerra ni Paz: comparaciones internacionales de niños y jóvenes en violencia armada organizada. Rio de Janeiro: COAV, 2005. P. 187-205.

CARRARA, Sergio et al. Curso de Especialização em Gênero e Sexualidade v. 4 e 5. Rio de Janeiro: CEPESC; Brasília: SPM, 2010.

CHÁVEZ, Francisco; MORALES, Melby. La Autoestima en Educandos con Tendencia Homosexual. 2002. 97 f. Monografía (Licenciatura en Ciencias de la Educación) - Universidad de El Salvador, San Salvador, 2002.

CONTRERAS, Juan; LÓPEZ, Alejandro. Actitudes delos Estudiantes de la Universidad de El Salvador, Sede Central, Tienen sobre la Homosexualidad durante el Periodo que Comprende del 3 de Febrero al 28 de Marzo de 2014. 2014. 88 f. Informe Final (Maestría en Servicios Integrales en Salud Sexual y Reproductiva) - Universidad de El Salvador, San Salvador, 2014.

CRUZ, Yenny; SÁNCHEZ, Reyna; AZCUÑAGA, Karen. ¿Son Aceptados los Homosexuales en su Ambiente Laboral? 1999. 54 f. Monografía (Comunicación y Periodismo) - Universidad Centroamericana 'José Simeón Cañas', San Salvador, 1999.

DE LEÓN, Wendy; MELGAR, Daniel; VELÁSQUEZ, René. La Prohibición del Matrimonio entre Personas del Mismo Sexo Regulado por el Artículo 11 del Código de Familia, que Violenta Principios y Garantías Constitucionales en El Salvador. 2011. 288 f. Monografía (Licenciatura en Ciencias Jurídicas) -Universidad de El Salvador, San Salvador, 2011.

DÍAZ, Zulma; VEGA, Sofía. La Violación de los Derechos Humanos a los Internos Homosexuales del Centro Penal de Sensuntepeque. 2007. 111 f. Monografía (Licenciatura en Ciencias Jurídicas) - Universidad de El Salvador, San Salvador, 2007.

1394 Educação \& Realidade, Porto Alegre, v. 42, n. 4, p. 1375-1397, oct./dic. 2017. 
EL SALVADOR. Ministerio de Educación. Resultados de la Información Estadística de Instituciones de Educación Superior 2013. San Salvador: MINED, 2014.

EL SALVADOR. Ministerio de Salud. Lineamientos Técnicos para el Abordaje en Salud de las Personas Lesbianas, Gays, Bisexuales y Transgénero. San Salvador: MINSAL, 2012.

FOUCAULT, Michel. A Arqueologia do Saber. Petrópolis: Editora Vozes, 1972.

FOUCAULT, Michel. Microfísica do Poder. Rio de Janeiro: Edições Graal, 1993.

GARAY, Ana; SALGUERO, Fátima; SEGOVIA, Sandra. Caracterización Psicológica de las Mujeres Lesbianas del Grupo Renacer de la Luna que se Reúne en la Asociación Entre Amigos de El Salvador. 2002. 174 f. Monografía (Psicología) Universidad de El Salvador, San Salvador, 2002.

GARCÍA, Guillermo; NAJARRO, Guillermo; RAMOS, Carlos. Relación entre los Recursos Cognitivos y la Tolerancia Hacia la Homosexualidad. 2014. 46 f. Monografía (Psicología) - Universidad Centroamericana 'José Simeón Cañas', San Salvador, 2014.

GIRÓ, Ada; MARTÍNEZ, Henry. Dogmatismo y Homofobia en Jóvenes de la Zona Metropolitana de San Salvador. 2012. 39 f. Monografía (Psicología) - Universidad Centroamericana 'José Simeón Cañas', San Salvador, 2012.

GIRÓN, Luz; TOLEDO, María; TORRES, Jeanny. Factores Psicosociales que Determinaron el Cambio de Identidad Sexual en Personas del Sexo Masculino entre las Edades de 16 a 30 Años de Edad Cronológica que Laboran en Salones de Belleza de la Ciudad de Santa Ana. 2007. 127 f. Monografía (Psicología) - Universidad de El Salvador, Santa Ana, 2007.

GOFFMAN, Erving. Estigma - Notas sobre a Manipulação da Identidade Deteriorada. Rio de Janeiro: Editora Guanabara, 1988.

GÓMEZ, Nicole; MARCENARO, Lorena; MEZA, Karla. Discriminación Sutil por Parte de Jóvenes Universitarios del Área Metropolitana de San Salvador Hacia la Homosexualidad. 2013. 44 f. Monografía (Psicología) - Universidad Centroamericana 'José Simeón Cañas', Antiguo Cuscatlán, 2013.

GUERRA, Giselle; ECHEVERRÍA, Dayma; BOMBINO, Yesisei. Estudios de Género y Sexualidad en Cuba: avances y retos desde el compromiso. In: VALCUENDE, José; MACARRO, María; ALARCÓN, Rubio (Coord.). Diversidad Sexual en Iberoamérica. Sevilla: Aconcagua, 2013. P. 197-211.

GUERRERO, Oscar Efrén; SUTACHAN, Helena Alexandra. 'En Colombia se Puede ser...': indagaciones sobre la producción de lo LGBT desde la academia. Nuevos Nómadas, Bogotá, n. 37, p. 219-229, oct. 2012.

GUZMÁN, Ana; MERLOS, Yeny. Factores Psicosociales que Inciden en la Homosexualidad de las Personas Inscritas en la 'Asociación Entre Amigos' de la Ciudad de San Salvador. 2006. 44 f. Monografía (Psicología) - Universidad de Oriente, San Miguel, 2006.

HERNÁNDEZ, Ada; VENTURA, Mónica. Estudio Jurídico-Doctrinario de la Aplicación Efectiva de los Derechos Constitucionales, Contemplados en la Legislación de Familia, a las Parejas Homosexuales Migueleñas, en el 2000-2001. 2002. 207 f. Monografía (Licenciatura en Ciencias Jurídicas) - Universidad de El Salvador, San Miguel, 2002.

HERNÁNDEZ, Dagoberto; HERNÁNDEZ, Miguel. Patrones Culturales que Discriminan a las Minorías de Homosexuales Hombres en el Empleo Informal de la Economía en el Área Metropolitana. 1998. 113 f. Informe Final (Maestría en Derechos Humanos y Educación para la Paz) - Universidad de El Salvador, San Salvador, 1998.

Educação \& Realidade, Porto Alegre, v. 42, n. 4, p. 1375-1397, oct./dic. 2017. 
HERNÁNDEZ, Porfirio. Los Estudios sobre Diversidad Sexual en el PUEG. In: CAREAGA, Gloria; CRUZ, Salvador. Sexualidades Diversas: aproximaciones para su análisis. México D. F.: Porrúa, 2004. P. 21- 34.

JIMÉNEZ, José. Temáticas en Construcción: el desarrollo de los estudios LGBT en Costa Rica, 1980-2013. Cuadernos Inter'c·a'mbio sobre Centroamérica y el Caribe, San José, v. 11, n. 2, p. 91-116, jul./dic. 2014.

LARA-MARTÍNEZ, Rafael. Indígena, Cuerpo y Sexualidad en la Literatura Salvadoreña. Soyapango: Editorial Universidad Don Bosco, 2012.

LÓPEZ, Anita; NAVES, Nelly. El impacto psicológico en el autoconcepto de las personas que han revelado su orientación homosexual a su familia. San Salvador: UCA, 2006. 67 p. Psicología, Universidad Centroamericana "José Simeón Cañas", San Salvador, 2006.

LÓPEZ, Mirna; MARTÍNEZ, Karla; UMAÑA, Yanet. Vivencias Homosexuales y Discriminación que Sufren las Adolescentes de 15 a 19 años, que se Encuentran Dentro del Centro Infantil de Protección Inmediata, de Marzo a Septiembre, 2012. 2012. 114 f. Monografía (Salud Materno Infantil) - Universidad de El Salvador, San Salvador, 2012

MARROQUÍN, Karen. Estudio Jurídico del Matrimonio entre Personas del Mismo Sexo a la Luz del Derecho Comparado. 2012. 83 f. Monografía (Licenciatura en Ciencias Jurídicas) - Universidad Francisco Gavidia, San Salvador, 2012.

MARTÍNEZ, Carlos; REYES, Ivette. El Impacto en la Sociedad Salvadoreña y Comunidad Homosexual a partir de la Propuesta de Reforma del Artículo 32 de la Constitución de la República, en Relación con el Reconocimiento del Matrimonio entre un Hombre y una Mujer 'así nacidos'. 2008. 129 f. Monografía (Licenciatura en Ciencias Jurídicas) - Universidad de El Salvador, San Salvador, 2008.

MEJÍA, Claudia; ORTIZ, Briseida; ROCA, Jacqueline. Identidad Sexual: la experiencia de mujeres lesbianas en el proceso de reconocimiento de su orientación sexual en un entorno familiar heterosexual - propuesta de intervención psicosocial para su desarrollo personal. 2013. 120 f. Monografía (Psicología) - Universidad de El Salvador, San Salvador, 2013.

MORÁN, Melida; PALMA, Víctor; TORRES, Balmore; ZELAYA, Jaime. Consecuencias Jurídicas y Morales de la Falta de Regulación de la Unión de Parejas del Mismo Sexo. 2011. 101 f. Monografía (Licenciatura en Ciencias Jurídicas) Universidad de El Salvador, Santa Ana, 2011

OCHOA, Xitlaly; LÓPEZ, Jaime. Estudio Jurídico e Institucional en Materia de Protección de los Derechos Humanos de la Población Lesbiana, Gay, Bisexual, Transexual, Transgénero, Travesti e Intersexual (LGBTI) de El Salvador. 2015. 201 f. Informe Final (Maestría en Derechos Humanos y Educación para la Paz) Universidad de El Salvador, San Salvador, 2015.

PARADA, Edgar; ESCOBAR, Manuel; TORRES, Carlos. La Discriminación Social en las Personas con Tendencia Homosexual. 2008. 66 f. Monografía (Psicología) - Universidad Evangélica de El Salvador, San Salvador, 2008.

PEW RESERCH CENTER. The Global Divide on Homosexuality: grearter acceptance in more secular and affluent countries. Washington: PewResearchCenter, 2013.

PISCITELLI, Adriana. Interseccionalidades, Categorias de Articulação e Experiências de Migrantes Brasileiras. Sociedade e Cultura, Goiânia, v. 11, n. 2, p. 263-274. 2008

1396 Educação \& Realidade, Porto Alegre, v. 42, n. 4, p. 1375-1397, oct./dic. 2017. 
REALEGEÑO, Ronald; VALLDEPERAS, Luis. Aplicación de Psicoterapia Individual a Homosexuales a Fin de Mejorar su Calidad de Vida. 2007. 94 f. Monografía (Psicología) - Universidad Modular Abierta, San Salvador, 2007.

RÍOS, Marina. La Identidad Personal de las Mujeres Lesbianas del Colectivo Las Desclosetadas, Municipio de San Salvador 2010 (Estudio de Caso). 2011. 70 f. Monografía (Trabajo Social) - Universidad Pedagógica de El Salvador, San Salvador, 2011.

ROSALES, Claudia; SÁNCHEZ, Rina; SEGOVIA, Johana. Influencia de las Variables Sociodemográficas en la Actitud Hacia la Homosexualidad, de los Estudiantes de la Universidad de Oriente. 2011. 64 f. Monografía (Psicología) - Universidad de Oriente, San Miguel, 2011.

RUBIN, Gayle. Reflexionando sobre el Sexo: notas para una teoría radical de la sexualidad. In: VANCE, Carole. Placer y Peligro: explorando la sexualidad femenina. Madrid: Revolución, 1989. P. 113-190.

SACA, Erika; VÁSQUEZ, Ana; ÁLVAREZ, Orlando. Historia de Vida de una Mujer Transexual. 2006. 54 f. Monografía (Comunicación Social) - Escuela de Comunicación Mónica Herrera, Santa Tecla, 2006.

VILLATORO, Mario; QUINTERO, Rosa. El Sida y su Incidencia en el Cambio de Actitudes Hacia la Práctica Homosexual de la Población Masculina en la Ciudad de San Salvador. 1988. 84 f. Monografía (Psicología) - Universidad Francisco Gavidia, San Salvador, 1988.

Amaral Palevi Gómez Arévalo es doctor en Estudios Internacionales en Paz, Conflictos y Desarrollo. Con experiencia profesional en la gestión de proyectos para el desarrollo con énfasis en trabajo comunitario, educativo, escolar y docencia universitaria. Sus líneas de investigación son Estudios de Paz, Violencias y Estudios LGBT.

E-mail: amaral.palevi@gmail.com 